\title{
Electrode Design for Capacitive Sensors
}

\author{
Hubert Zangl \\ Institute of Electrical Measurement and Measurement Signal Processing \\ Graz University of Technology \\ Kopernikusgasse 24/IV,8010 Graz, Austria
}

\section{Introduction}

Capacitive sensing is successfully used for measurement tasks in various areas, ranging e.g. from position measurement in the sub-nanometer range like in atomic force microscopy (e.g. [1]) over the detection of lowest concentrations of chemical substances in lab on chip systems ([2]) to position measurement in the touch screens of mobile phones. For capacitive sensors, high precision measurement techniques have been developed over the past years. Commercial integrated circuits permit determination of capacitances with a resolution of a few aF (e.g [3, 4]) and even measurements in the $\mathrm{aF}$ range with rates of more than ten thousand measurements per second have been reported [5].

However, a capacitive sensor does not only require an accurate electronic evaluation circuitry. Also, the electrode topology plays a major role for the performance of a sensor. Figure 1 shows an example problem. Here, there permittivity of a small object inside a tube is of interest. Electrodes may be placed anywhere around the circumference of the pipe (accessible boundary) but not inside of the pipe. The question is now, where electrodes should be placed and what dimensions would be optimal for the determination of the object's permittivity

Frequently, the initial electrode design for capacitive sensors is chosen according to the knowledge of an expert and optimization is done in time consuming experiments. An alternative approach is given by model based optimization using field computation software and standard optimization techniques [6,7]. However, this is still a complex task as the location and size of electrodes span a huge parameter space. In the following, a fast method for the design of electrode topologies which aims to provide a good signal to noise ratio is presented.

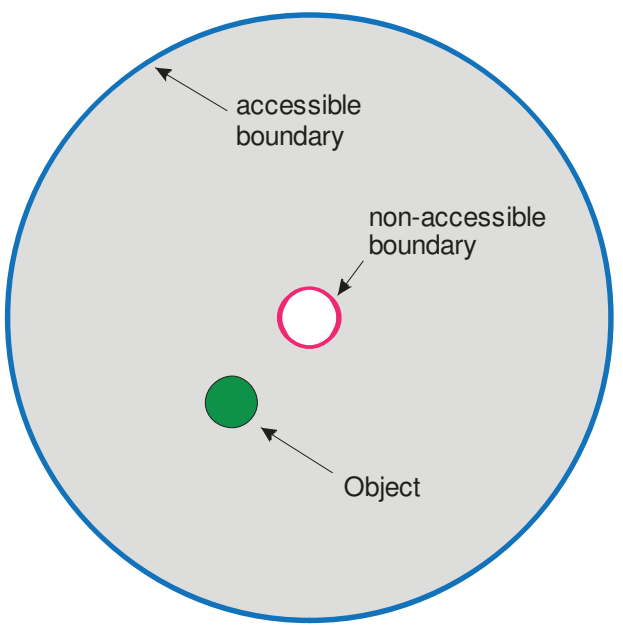

Figure 1: Example problem: The accessible boundary can be used to place electrodes for capacitive sensing. The parameter of interest is the permittivity of the object. 


\section{Objective Functions and Optimality Criteria for Sensor Design}

Before an optimization can start, it is important to clearly understand what optimilty means in a certain application and how it should be described mathematically. This leads to the problem to make an appropriate choice for the optimality criterion, i.e. we need a decision what we consider a good design. For sensors it is commonly accepted that a good sensor has a low mean square error, although this may not be appropriate for any situation. Sometimes, linearity, monotonicity, repeatability and so on may be more important than a low mean square error. Once we have decided for the optimality criterion, an optimization algorithm tries to find the parameter vector $\xi^{*}$ to fulfill the optimality condition:

$$
\xi^{*}=\arg \min \Psi(\xi)
$$

The optimality condition (1) requires an objective function $\Psi$. Many different ones are proposed in literature. Reviews can be found e.g. in [8], [9], and [10]. Most of the criteria found in literature are based on the Fisher information I. These criteria do not require particular estimators as they minimize certain properties of the dispersion matrix D, which is in essence the Cramer Rao lower bound (CRLB). In the multi-dimensional case, i.e. when more than one quantity is measured, different properties of the dispersion matrix D may be of interest. The most common D-criterion and corresponds to a minimization of the volume of the uncertainty region, the Acriterion minimizes the averaged variance of the states. The E-criterion minimizes the largest eigenvalue of D corresponding to the largest main axis of the uncertainty ellipsoid. If the sensors are biased, a similar criterion can be used for the mean square error rather than the covariance [6].

In order to perform an optimization it is usually necessary to find a parametric description of all feasible electrode designs. For instance, a number of circular electrodes, described by their center origins and radii could be used. An optimization procedure for capacitive sensors designs could now be applied to vary these parameters in order to find a good arrangement. However, this is a rather complex procedure, and requires a good parameterization of the electrode designs. As an advantage, the procedure really aims to improve the desired property of the sensor. Due to the complexity of this approach an alternative approach is suggested in the following. Although the suggested method does not directly optimize the sensors performance, it can be assumed that the obtained sensor performace will also be good. The novel method offers the advantage that a parametric description of possible electrode topologies is not required. Furthermore, the evaluation is comparatively fast. In order to further improve such designs, they may still be used as starting geometries for a consecutive optimization based on one of the above criteria for direct sensor optimization.

\section{Capacitive Sensor Design by Maximum Uniform Sensitivity}

The uncertainty that causes inaccurate measurement results has different origins. Some nuisance parameters are to due manufacturing tolerances, e.g. deviations in geometry or material properties. Another source of uncertainty is caused by noise or disturbers. In order to keep these influences low, the sensitivity of the sensor,

$$
S=\frac{\partial I}{\partial p}
$$

i.e. the change of the displacement current as a consequence of a change of the measurement quantity should be high, whereas cross-sensitivies (i.e. a change of the displacement current for other reasons that a change of the parameter of interest) should be low. Consequently, an optimization of the sensitivity of a sensor does not necessarily mean an improvement of the senor performance, as the objective function is not e.g. the mean square error. However, in certain situations it is reasonable to assume a strong correlation between high sensitivity and low mean square error. This is the case when the cross sensitivities are less affected by the electrode design. In such situations an optimization of the sensitivity will also improve e.g. the mean square error.

Considering capacitive sensors we find that the change of the displacement current with respect to a parameter of interest also scales with the applied voltage. Therefore, it is more useful to consider the change of ratio of displacement current and applied potential (change of susceptance) with respect to a change of the parameter of interest. This parameter may be considered as a local relative sensitivity and is given by 


$$
S_{l r}=\frac{\frac{\partial I}{\partial p}}{U}
$$

Theoretically, a capacitive sensor could use an infinite number of tiny electrodes, impress voltage on these electrodes and measure the displacement currents. The determination of all inter-electrode capacitances would be impossible, as the number of combinations is infinite. However, only certain voltage patterns will provide a high sensitivity with respect to the parameters of interest. In this sense, it is reasonable to search for voltage patterns that maximize the local relative sensitivity. As the relative sensitivity according to (3) is defined for each point on the boundary, it is not a scalar value and thus directly applicable as an objective function. A scalar objective function based on the relative sensitivity could be e.g. the maximum, the mean or the median. The following method maximizes the uniform relative sensitivity.

\section{Design Method for Electrode Topologies}

With the Finite Element Method it is possible to transform the partial differential equation $\nabla \cdot\left(\varepsilon_{0} \varepsilon_{r} \nabla U\right)=0$ describing the electric field into a linear equation system of the form

$$
K U=0
$$

where $K$ is the so called stiffness matrix. $K$ depends only on the geometry and the material properties. $\mathrm{U}$ represents the electric potential at certain discrete locations, which are obtained by a discretization of a geometry into small elements, e.g. tetrahedrons. In order to consider Dirichlet boundary conditions, (i.e. assigned potentials) $K$ is modified such that all lines corresponding to nodes where the potential is assigned are replaced with ones in the main diagonal. With the modified stiffness matrix $\tilde{K}$ the potentials are obtained by solving

$$
\tilde{K} U=X
$$

where $X$ contains the assigned electrode potentials. The original stiffness matrix can now be used to calculate the surface displacement currents by

$$
K U=I
$$

If the geometry or a material property changes, this leads to a modification of the stiffness matrix. Consequently, the change of the displacement current can be determined.

With above definitions, we can use the following eigenvalue problem to find the boundary voltage distribution with maximum uniform sensitivity (all local relative sensitivities are equal):

$$
\left[\frac{\partial K}{\partial p} \tilde{K}^{-1}-K \tilde{K}^{-1} \frac{\partial \tilde{K}}{\partial p} \tilde{K}^{-1}\right] X=\frac{\partial I}{\partial p}=\lambda X
$$

The vector $X$ represents the boundary voltage distribution. The parameter $p$ represents the quantity of interest. The use of the obtained distribution for an electrode design would lead to a fairly high number of independent electrodes. It is now suggested to connect electrodes that have similar potential and further neglect those locations with very low potentials. In this sense the fine discretization of the boundary is replaced by a small number of electrodes. For this purpose it is only required to use a threshold and then the electrode topologies approximating the optimal distribution(s) with a small number of discrete electrodes.

\section{Application of the Method}

Figure 1 in section I shows an example problem in 2D. In this case, the entire circumference of the problem domain is accessible for electrode placement, whereas the hole in the middle of the problem domain is not. The parameter of interest is the permittivity of the shown object. Figure 2 shows two different voltage patterns that provide the highest 
uniform relative sensitivity, which obtained by solving (7). Although the two distributions are fairly different, they both achive good sensitivity towards the permittivity of the small object. Figure 3 shows the voltage pattern after a discretization to a two electrodes and ground. The relative sensitivity $S_{\text {loc }}$ changes from to $0.29 \Omega$ to $0.24 \Omega$ indicating that a high sensitivity of the electrode topology is maintained. Figure 4 shows the impact of discretization in detail. With the eigenvalue methods, a theoretic, continuous voltage pattern is obtained for each eigenvalue. If the voltage pattern was applied, then the maximum uniform relative sensitivity would be obtained. However, in practice only a few electrodes can be used. Therefore, the continuous distribution is approximated with two electrodes. Despite the drastic discretization, the sensitivity distribution is only moderately changed and the maximum sensitivity only drops by about $10 \%$. This shows that the method - although it is continuous in principle - can be used to find a small number of electrodes. The method is not restricted to permittivity variations and can also be applied to find electrode topologies for, e.g. distance or level sensors.
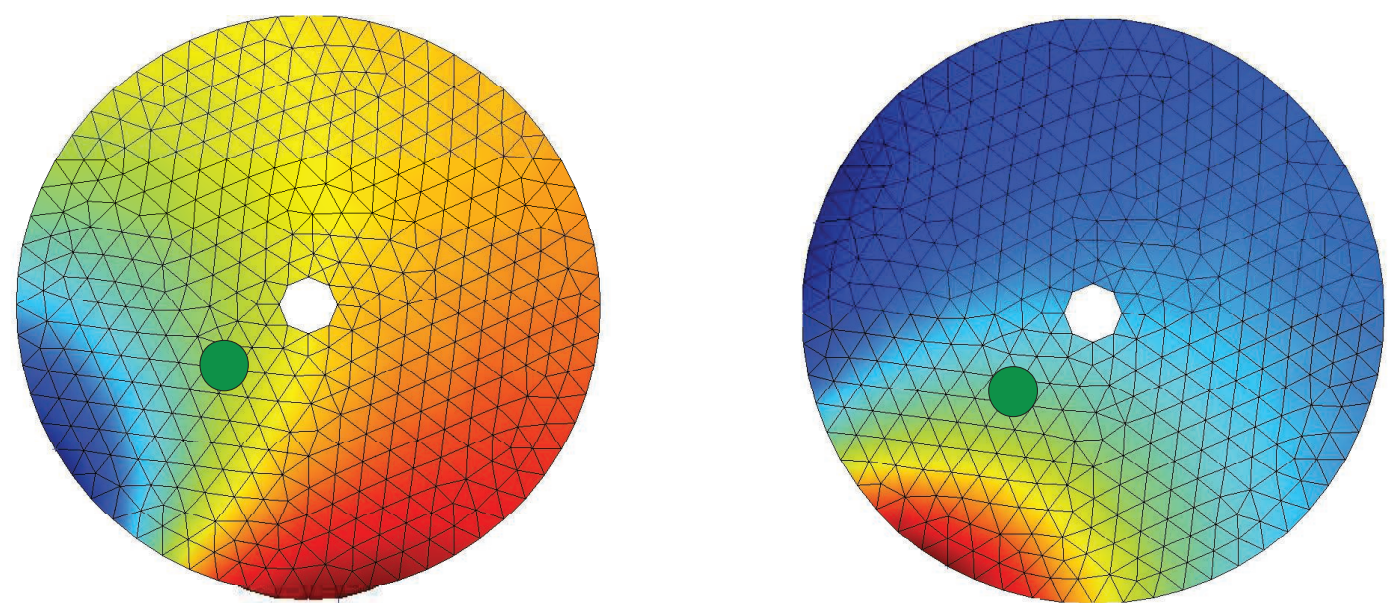

Figure 2: Color plot of voltage distributions for the two surface voltage distributions with highest uniform sensitivity.
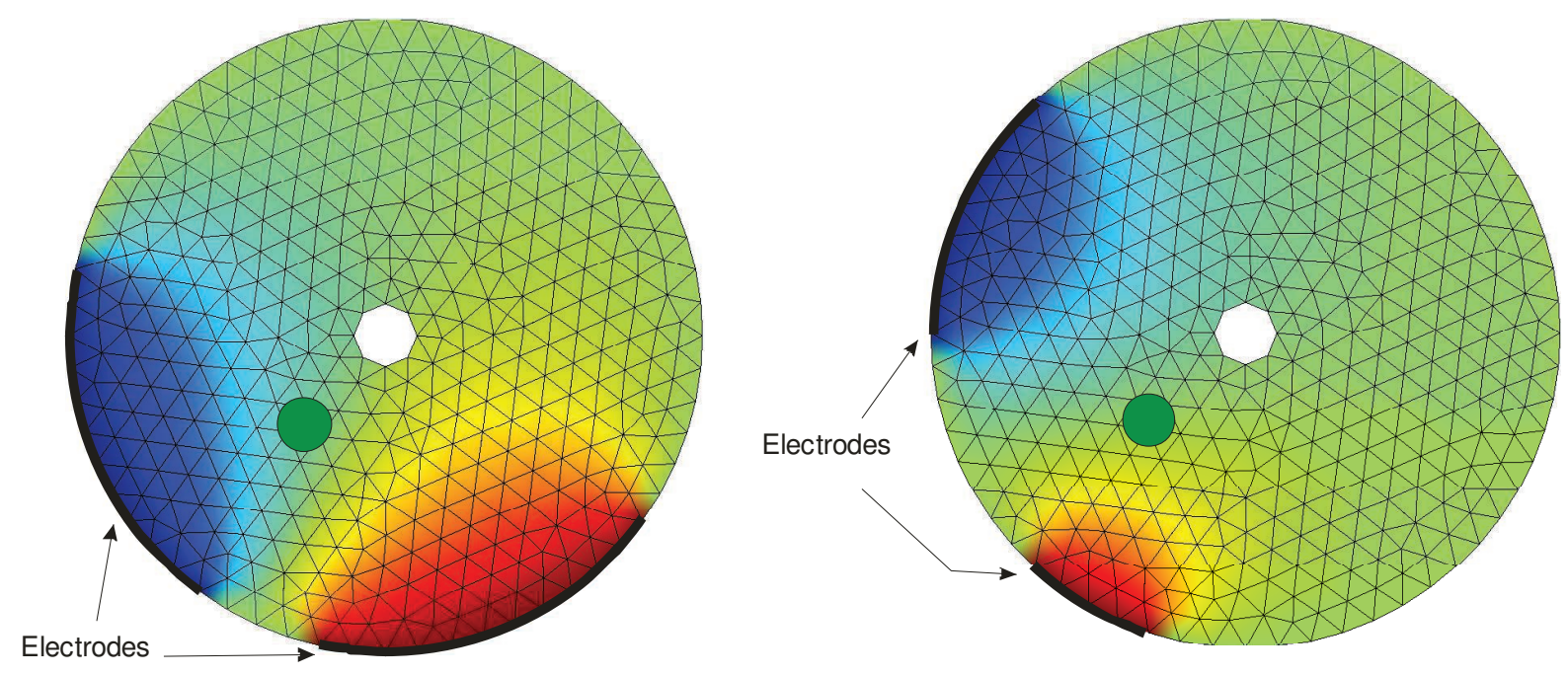

Figure 3: Color plot of voltage distributions for the two surface voltage distributions with highest uniform sensitivity (ref. 2) after a reduction to two electrodes. The relative sensitivity is only slightly reduced. Combining the electrode configurations for both voltage distributions leads to the final electrode geometry and excitation sequence. 


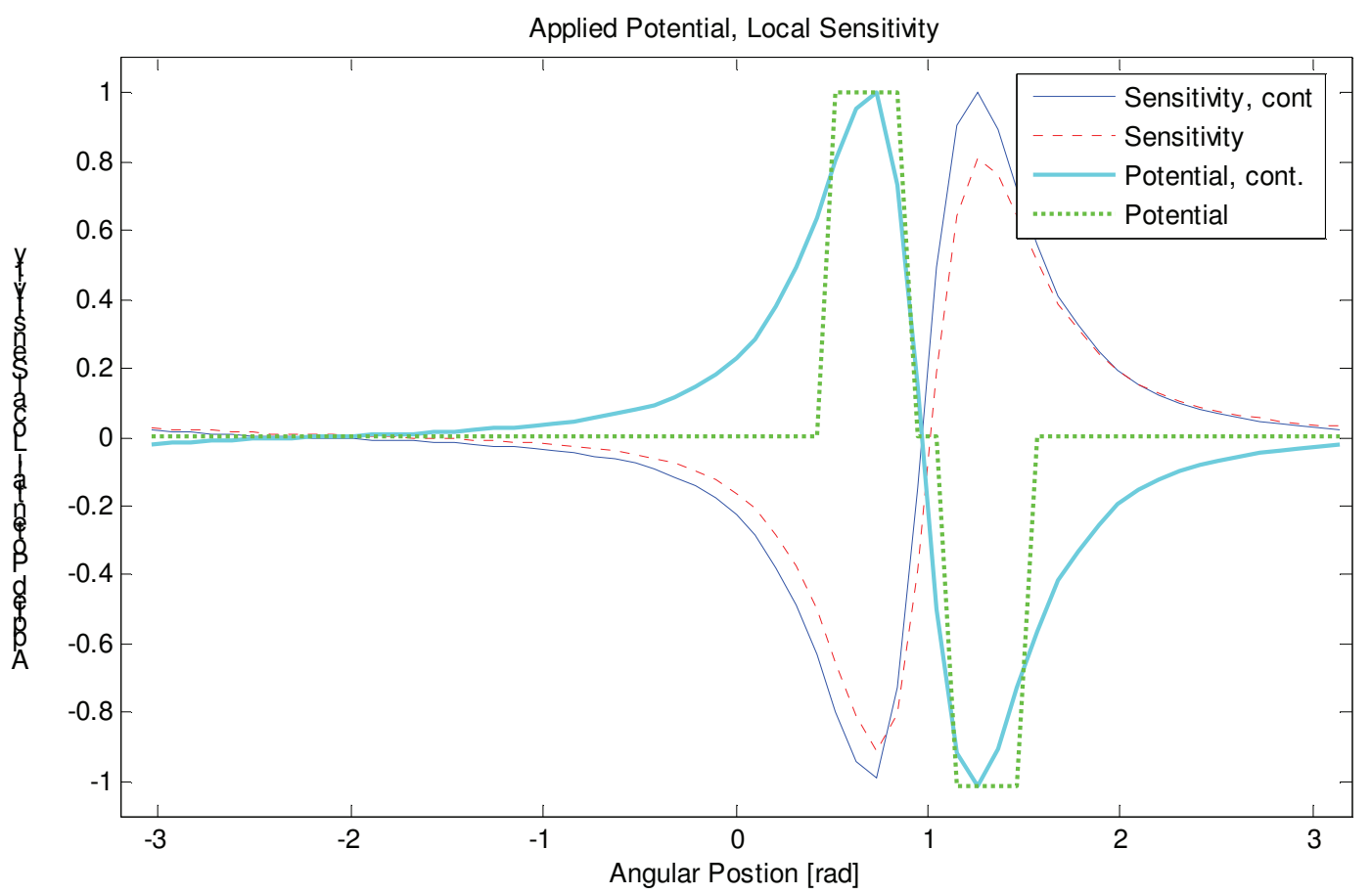

Figure 4: Electrode Discretization: With the eigenvalue methods, a theoretic, continuous voltage pattern is obtained for each eigenvalue. The figure shows the distribution for a negative eigenvalue (i.e. the signal decreases when the permittivity increases). If the voltage pattern was applied, then the maximum uniform relative sensitivity would be obtained. However, in practice only a few electrodes can be used. Therefore, the continuous distribution (cyan) is approximated with two electrodes (green, dashed; +1: Electrode 1, -1: Electrode 2). Despite the drastic discretization, the sensitivity distribution is only moderately changed and the maximum only drops by about $10 \%$. This shows that the method can also be used to place a small number of electrodes.

\section{Conclusion}

A fast method for electrode design of capacitive sensors is suggested. Instead of the mean square error, the objective function is based on the local relative sensitivity. Therefore, the method is particular useful whenever the mean square error strongly decreases with increasing local relative sensitivity. In other situation it may be used to obtain an initial electrode configuration for more complex optimization procedures. The method is comparatively fast and has the major advantage, that no parametric description of possible electrode configurations is needed.

\section{Acknowledgment}

This work was partially funded by the Austrian Science Fund under grant P21855.

\section{References}

[1] I. A. Mahmood, S. Moheimani, K. Liu, Tracking control of a nanopositioner using complementary sensors, IEEE Transactions on Nanotechnology 8 (1) (2009) 55-65. doi:10.1109/TNANO.2008.2005183.

[2] S.-W. Wang, C.-H. Lin, Y.-S. Yang, M. S.-C. Lu, A cmos capacitive dopamine sensor with sub-nm detection resolution, in: Proc. IEEE Sensors, 2009, pp. 400-404. doi:10.1109/ICSENS.2009.5398250.

[3] http://www.cypress.com/capsense/ (June 2008).

[4] http://www.analog.com/static/imported-files/data_sheets/AD7747.pdf (February 2007).

[5] R. Nojdelov, S. Nihtianov, Capacitive-sensor interface with high accuracy and stability, IEEE Transactions on Instrumentation and Measurement 58 (5) (2009) 1633-1639. doi:10.1109/TIM.2009.2012957.

[6] H. Zangl and G. Steiner, “Optimal design of multi-parameter multi-sensor systems," IEEE Transactions on Instrumentation and Measurement, vol. 57, pp. 1484-1491, July 2008.

[7] X. Hu and W. Yang, 'Planar capacitive sensors - designs and applications," Sensor Review, vol. 30, pp. 24-39 
[8] R.H. Myers and D.C. Montgomery. Response Surface Methodology. Wiley Series in Probability and Statistics. John Wiley \& Sons, Inc., New York, 2nd edition, 2002.

[9] V. Fedorov and P. Hackl. Model-Oriented Design of Experiments, volume 125 of Lecture Notes in Statistics. Springer, NY, USA, 1997.

[10] S.D. Silvey. Optimal Design. Chapman and Hall, London, 1980. 